\title{
Séquences d'évolution morphodynamique des barres intertidales d'une plage macrotidale : l'exemple d'Omaha beach (Normandie, France)
}

\section{Morphodynamical evolution sequences of intertidal bars on a macrotidal beach: case study of Omaha beach (Normandy, France)}

\author{
Alexis Stépanian *, Franck Levoy \\ Unité M2C, Morphodynamique continentale et côtière, UMR CNRS 6143, 24, rue des tilleuls, 14000 Caen, France.
}

Reçu le 1 juin 2002 ; révisé et accepté le 4 octobre 2002

\begin{abstract}
Résumé
Cet article traite de la morphodynamique des barres intertidales présentes sur la plage macrotidale Omaha beach située sur la côte du Calvados en Normandie. Durant deux années, ces systèmes, localement dénommés plages à barres et bâches, ont fait l'objet d'un suivi topographique à un pas de temps mensuel défini comme moyen-terme. L'analyse combinée de l'évolution morphologique et des enregistrements des conditions de forçage, vagues et marée sur la basse plage, montre l'existence de séquences d'évolution morphodynamique comprenant chacune une phase d'accrétion et une phase de destruction des barres sableuses. Afin de les distinguer, un critère énergétique, basé sur l'intensité de l'agitation, est utilisé. Les phases d'accrétion correspondent à de longues périodes de faible agitation (hauteur significative de la houle, $\mathrm{H}_{\mathrm{s}}<1 \mathrm{~m}$ ) pendant lesquelles les barres intertidales se construisent et migrent vers le haut de plage à une vitesse atteignant $0,4 \mathrm{~m}$ par jour. A l'inverse, les phases de destruction résultent d'augmentations rapides du niveau d'énergie des houles $\left(\mathrm{H}_{\mathrm{s}}>1 \mathrm{~m}\right)$ lors de tempêtes, dont l'action érosive est modulée par le niveau tidal et la morphologie antécédente. À moyen terme, l'ensemble des résultats montre des disparités de comportement morphodynamique de l'estran, les barres étant plus mobiles sur la partie supérieure du profil que sur la partie inférieure. Une dynamique de chenaux de drainage est mise en évidence. C'est un facteur de déstabilisation des barres qui fragilise leur résistance à l'action des tempêtes. De plus, la présence des barres et leur évolution morphologique est la traduction topographique de réorganisations d'un stock sableux intertidal stable à moyen terme.
\end{abstract}

(C) 2003 Éditions scientifiques et médicales Elsevier SAS. Tous droits réservés.

\begin{abstract}
The present paper deals with the morphodynamics of intertidal bars on Omaha beach, a macrotidal beach located along the Normandy coastline. During two years (defined here as medium-term time-scale), a monthly topographic monitoring has been conducted on this beach. An analysis of the morphological evolution of the ridge and runnel systems is performed and correlated to the forcing conditions (waves and tide) recorded on the lower foreshore. Our results allow to distinguish some morphodynamical evolution sequences with one stage of accretion and one stage of destruction of the bars. In order to discriminate these two stages, an energetic criterion is used. Accretion stages correspond to long periods of fair weather conditions (significant wave height, $\mathrm{H}_{\mathrm{s}}<1 \mathrm{~m}$ ), when bars build up and migrate onshore with a rate under $0.4 \mathrm{~m}$ per day. On the opposite, destruction stages result from wave height increases during storms $\left(\mathrm{H}_{\mathrm{s}}>1 \mathrm{~m}\right)$. The destructive processes are modulated by tide level and pre-existing morphology. Our results show also a medium-term morphodynamic variability along the beach profile. Indeed, the upper beach bars are much more mobile than the lower beach ones. The drainage channel dynamic is also highlighted as a destabilizing factor which weakens the bar resistance to storm activity. Moreover, the construction and the evolution of intertidal bars correspond to short-term reorganisations of an intertidal sand volume which seems to be quite stable over a medium-term period.
\end{abstract}

(C) 2003 Éditions scientifiques et médicales Elsevier SAS. All rights reserved.

* Auteur correspondant.

Adresse e-mail : stepanian@geos.unicaen.fr (A. Stépanian). 
Mots clés : Morphodynamique ; Plage macrotidale ; Barres et bâches ; Normandie ; France

Keywords: Morphodynamics; Macrotidal beach; Ridges and runnels; Normandy; France

\section{Introduction}

Récemment, les études concernant les plages micro- et mésotidales ont largement amélioré la connaissance du comportement morphodynamique à moyen terme des barres sableuses (Lee et al., 1998 ; Shand et Bailey, 1999 ; Shand et al., 1999 ; Ruessink et Terwindt, 2000 ; Ruessink et al., 2000). En revanche, l'étude morphodynamique des barres des estrans macrotidaux reste marginale en raison de la complexité des interactions hydrodynamiques dans ces environnements mixtes où l'action de la houle est combinée à un fort impact de la marée. Pourtant, les plages macrotidales à barres sont particulièrement fréquentes sur les façades maritimes du nord-ouest de l'Europe. Elles sont présentes sur la côte belge (Voulgaris et al., 1998), la côte est de l'Irlande (Mulrennan, 1992), les côtes anglaises (King et Williams, 1949) et françaises. Ce type de plage est, en effet, une caractéristique majeure du littoral sableux français entre Dunkerque et la Baie de Somme (Corbau et al., 1994 ; Levoy et al., 1998 ; Masselink et Anthony, 2001), ainsi que localement sur les côtes du Calvados (Stépanian et al., 2001).

L'estran de ces plages est marqué transversalement par une alternance de crêtes et de sillons dont le nombre varie de deux à cinq entre les niveaux de pleines mers et de basses mers de vives-eaux. L'amplitude crête à creux de ces barres dépasse rarement $1 \mathrm{~m}$ et leur extension latérale est de l'ordre de quelques centaines de mètres. Elles sont délimitées par des chenaux de drainage, perpendiculaires ou obliques au trait de côte, par où s'évacue l'eau à la marée descendante. Cette morphologie intertidale, localement dénommée « barres et bâches ", correspond aux ridges and runnels définies par King et Williams (1949). Ces barres se développent sur des plages de sables fins à moyens, à pente faible, soumises à un marnage important et à une agitation résultante de l'action du vent sur un fetch limité. Il découle de cet ensemble de facteurs que les ridges and runnels ou barres et bâches sont spécifiques de plages macrotidales en bordure de mers semifermées : Manche, Mer d'Irlande et Mer du Nord, pour l'Europe.

Bien que les premières études portant sur ces corps sableux datent des années 40 en prévision du débarquement allié sur les côtes de Normandie, les processus physiques à l'origine de la formation de ces systèmes de barres restent à l'heure actuelle indéterminés (Short, 1999). Les principales hypothèses avancées dans la littérature font référence à l'action des processus de swash, qui tendent à augmenter localement la pente du profil de plage et à créer une barre comme sur les plages micro et mésotidales (King, 1972 ; Kroon, 1994). Une hypothèse de formation par convergence sédimentaire engendrée par l'interaction entre des ondes infra- gravitaires et les groupes de vagues incidentes, est également évoquée par Simmonds et al. (1995, 1996).

Les mesures de l'évolution topographique de l'estran à une échelle de temps mensuelle montrent que les barres bien développées sont généralement des formes résultantes de périodes de faible agitation (King, 1972 ; Mulrenann, 1992). Les mesures hydrodynamiques et de transport sédimentaire dans de telles conditions soulignent une migration des barres vers le haut de plage (vitesse $\leq 1 \mathrm{~m} \mathrm{j}^{-1}$ ) sous l'effet des processus de swash qui balayent l'estran bi-quotidiennement (Levoy et al., 1998 ; Voulgaris et al., 1998 ; Stépanian et al., 2001). Cependant, ces mesures ponctuelles restent insuffisantes pour conclure que l'action du swash est le moteur principal de la genèse et de la migration de ces morphologies. L'hypothèse d'une formation sous l'action d'ondes infragravitaires, étayée à l'aide de modélisations numériques, n'a pas été, pour le moment, validée par des mesures in situ.

L'action des tempêtes sur ces formes est également source de nombreuses interrogations. Toutefois, les études portant sur l'évolution de plages macrotidales à barres, à une échelle de temps mensuelle, montrent que les évènements de haute énergie ont tendance à lisser la morphologie de barres (King, 1972). La destruction complète de certaines barres sur le profil est parfois observée, mais il en subsiste toujours, notamment sur le bas estran. Mulrennan (1992) qualifie ces systèmes de barres « semi-permanentes ».

Cette note présente l'évolution morphologique de la plage macrotidale à barres d'Omaha beach (Calvados) à une échelle de temps mensuelle à annuelle, définie comme moyen terme. L'objectif est d'identifier les diverses morphologies de barres intertidales et de préciser leur localisation préférentielle sur l'estran. L'analyse combinée de l'évolution morphologique et des conditions hydrodynamiques permet d'appréhender les modes d'évolution morphodynamique des barres sableuses à l'échelle de temps choisie. L'investigation porte également sur l'identification de seuils énergétiques de l'agitation, discriminant des périodes de construction et de destruction des barres.

\section{Site d'étude et méthodologie}

Omaha beach est une plage de la côte du Calvados (France). Elle constitue une anse sableuse d'une longueur de $6 \mathrm{~km}$ située entre deux portions rocheuses du littoral Fig. 1. La plage sensu stricto est séparée, par un étroit cordon dunaire, d'un marais maritime accolé à la falaise fossile. Elle est située dans un environnement macrotidal où le marnage atteint 6,2 $\mathrm{m}$ en vive-eau moyenne. L'agitation, étroitement corrélée avec les caractéristiques des vents locaux, correspond de manière dominante à une «mer de vent ». La hauteur significative moyenne de la houle, mesurée au niveau des 


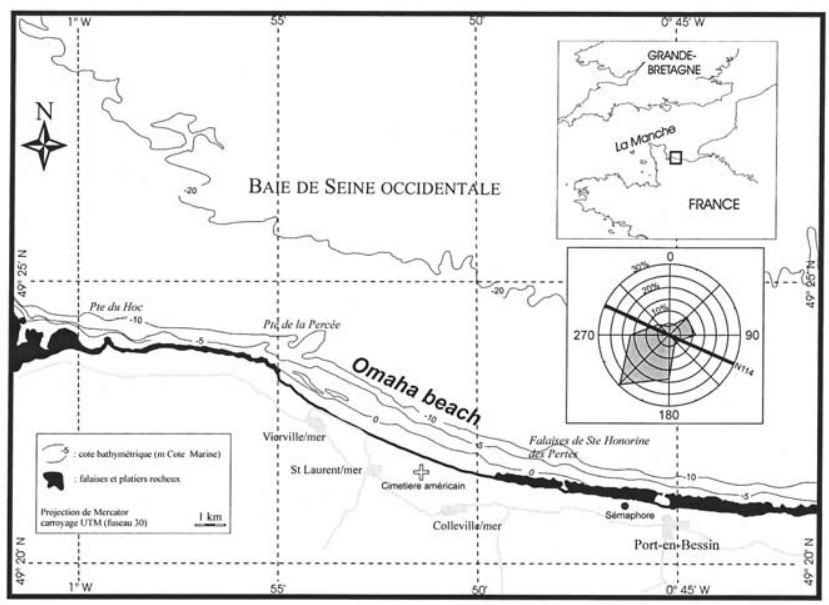

Fig. 1. Localisation de la plage d'Omaha beach sur la côte du Calvados. La rose des vents représente la distribution de la fréquence des directions de vents, au sémaphore de Port-en-Bessin pour la période février 1999-février 2001 (données Meteofrance).

Location of Omaha beach on the Calvados coast. The wind rose represents the wind direction frequency at Port-en-Bessin, from February 1999 to February 2001 (data property : Meteofrance).

basses mers de vives-eaux, est de $0,44 \mathrm{~m}$ pour une hauteur maximale de $3 \mathrm{~m}$ lors des tempêtes. Les périodes de pic de la houle associées à ces agitations, sont généralement de 4 à $5 \mathrm{~s}$ et atteignent $10 \mathrm{~s}$ en phase de haute énergie. Les houles en provenance du quadrant NW sont observées pendant $70 \%$ du temps. Elles sont prédominantes sur les houles de NE (30\%). Sur la période de mesures, les vents de terre sont plus fréquents que ceux en provenance du large.

Au cours du suivi, deux barres subtidales ont pu être identifiées sur le proche avant côte $(\tan \beta=0,014)$. La pente générale de la zone intertidale $(\tan \beta)$ est de l'ordre de 0,015 . Le profil transversal présente des systèmes de barres dont le nombre varie entre deux et cinq entre février 1999 et février 2001 Fig. 2. Ces barres sont des corps sableux tabulaires d'une extension latérale (longshore) de 200 à $400 \mathrm{~m}$, qui présentent parfois une légère obliquité, de quelques degrés, par rapport au trait de côte. Elles sont limitées longitudinalement par des chenaux de drainage sensiblement orthogonaux Fig. 2. Les sédiments superficiels sont des sables fins à moyens, homogènes et bien triés de médiane granulométrique moyenne $d_{50}=0,260 \mathrm{~mm}$. Le revers marin des barres présente généralement des lits plans, alors que les fonds des bâches sont couverts de figures sédimentaires variables en fonction des conditions hydrodynamiques (rides 2D, 3D, rides interférence et mégarides). Cette répartition générée par la dynamique des écoulements sur la morphologie de barres, est conforme aux observations de King (1972) sur des plages similaires.

La Fig. 3 présente un exemple de profil de plage type, ainsi que la zonation marégraphique utilisée pour l'étude des plages à marée. Ce découpage est basé sur une prédominance des processus hydrodynamiques : swash et déferlement sur la haute plage, déferlement sur la moyenne plage et vagues non déferlantes et courants de marée sur la basse plage (Wright et
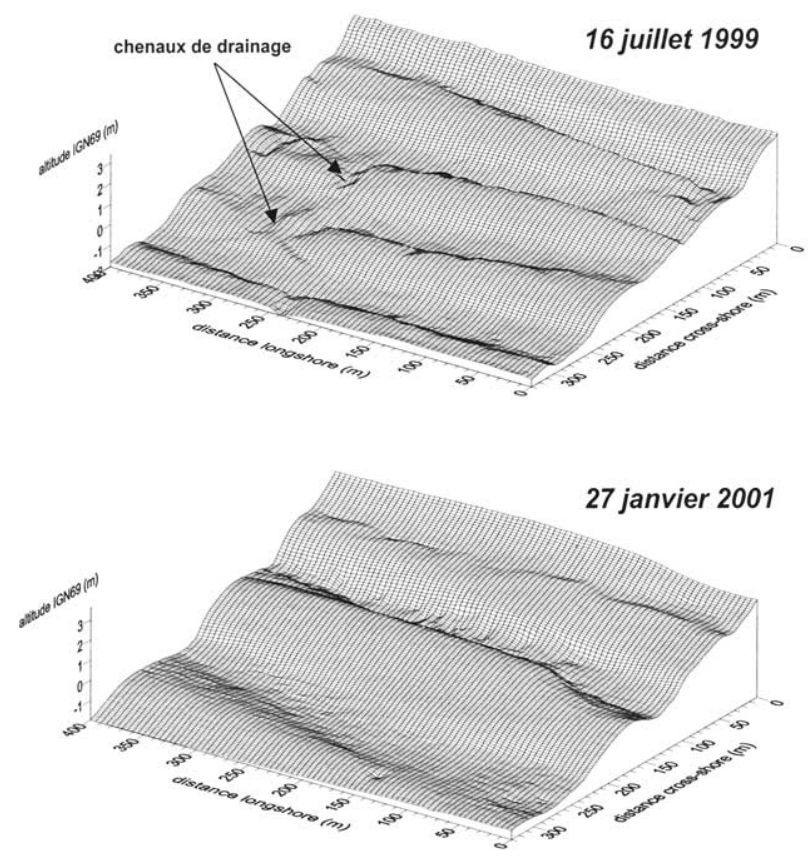

Fig. 2. Exemples de Modèles Numériques de Terrain d'Omaha beach. Ces deux situations montrent la variabilité du nombe de barres en fonction du temps et la dimension spatiale des chenaux de vidange.

Samples of Digital Elevation Model of Omaha beach - The two cases show the time variability of the bar quantity and different spatial dimensions of drainage channels.

al., 1982 ; Masselink, 1993 : Levoy et al., 2001). Il permet d'un point de vue pratique, de préciser la localisation des évolutions morphologiques sur le profil. L'altitude en cote marine $(\mathrm{CM})$ des niveaux moyens de marée est calculée à Port-en-Bessin (10 km à l'est d'Omaha beach) en fonction du niveau moyen de la mer $\mathrm{Z}_{0}(4,4 \mathrm{~m}$ C.M.), de l'amplitude de l'onde lunaire M2 (2,32 m) et de l'onde solaire S2 $(0,78 \mathrm{~m})$ (Hardisty, 1990). Ces altitudes sont ramenées, par la suite, au référentiel IGN69 (0 m C.M. = -4,05 m IGN69)

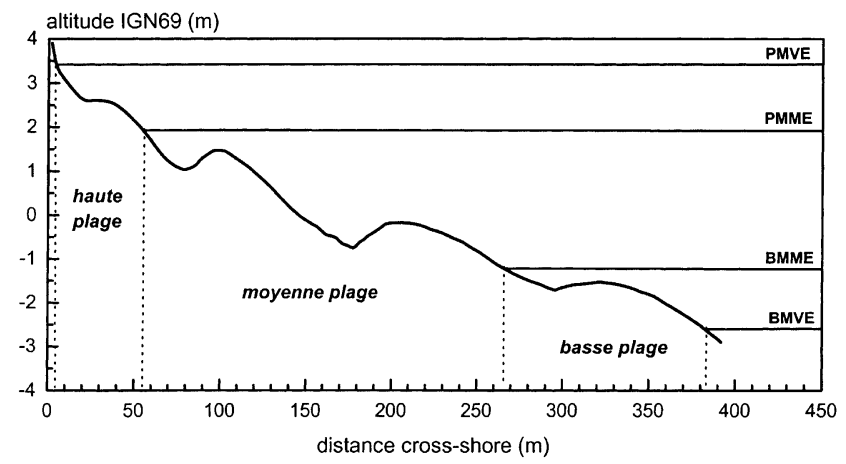

Fig. 3. Exemple de profil de plage d'Omaha beach et zonation en fonction des niveaux moyens de marée. $\mathrm{PMVE}=$ niveau moyen des pleines mers de vives-eaux $;$ PMME = niveau moyen des pleines mers de mortes-eaux ; $\mathrm{BMME}=$ niveau moyen des basses mers de mortes-eaux $; \mathrm{BMVE}=$ niveau moyen des basses mers de vives-eaux.

An Omaha beach profile with successive tidal zones. PMVE = mean level of spring high tides $; \mathrm{PMME}=$ mean level of neap high tides $; \mathrm{BMME}=$ mean level of neap low tides $; \mathrm{BMVE}=$ mean level of spring low tides. 
a) $\mathrm{H}_{\mathrm{s}}(\mathrm{m})$

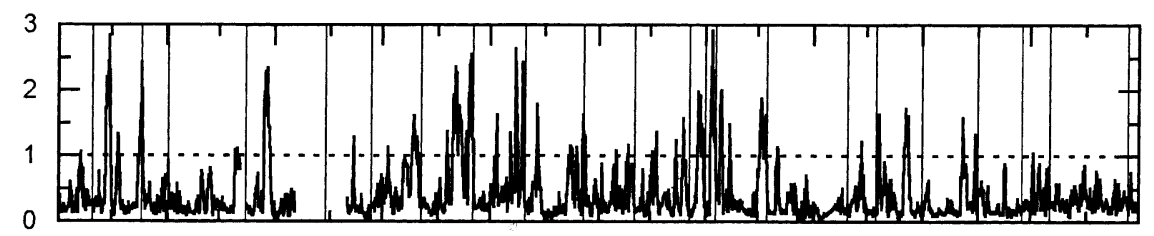

b) $T_{s}(s)$

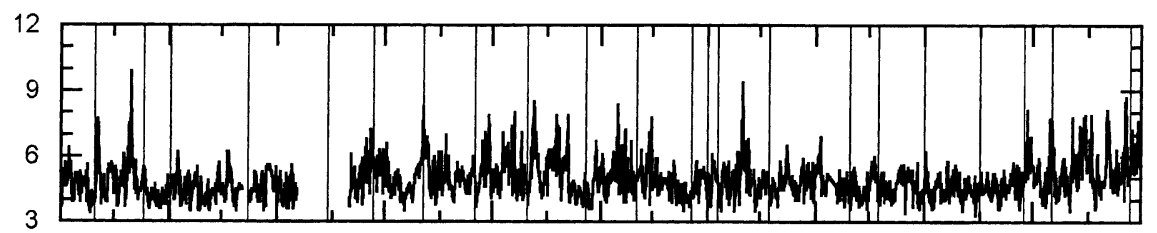

c) $\mathrm{H}_{\mathrm{s}} \mathrm{L}$
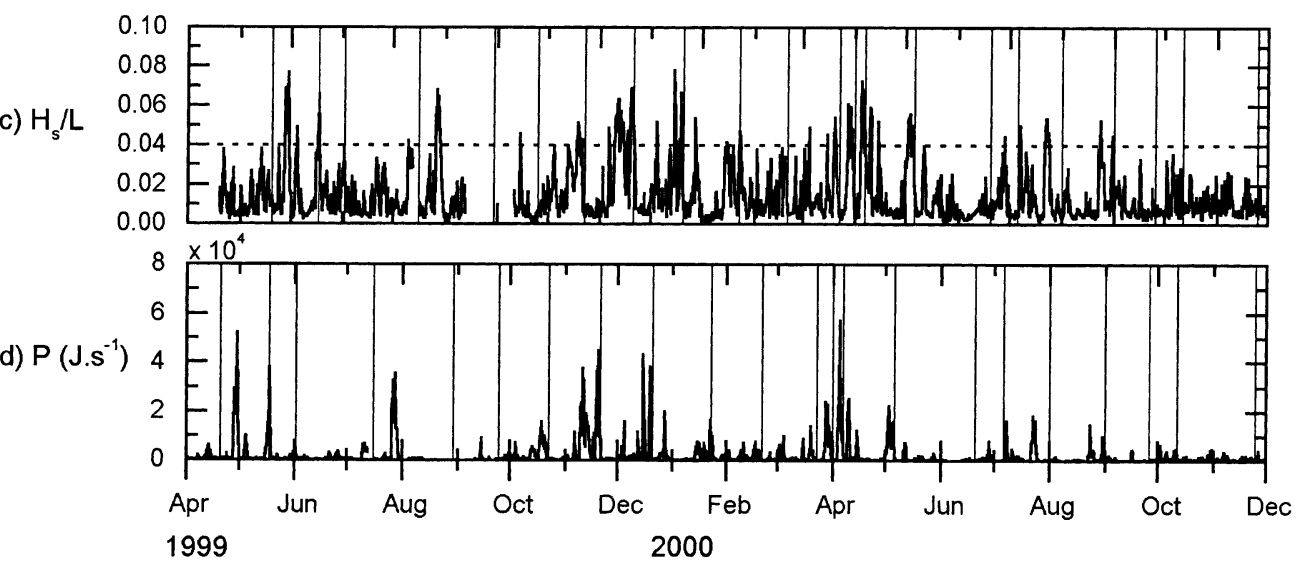

Fig. 4. Séries chronologiques des $\mathrm{H}_{\mathrm{s}}(\mathrm{a}), \mathrm{T}_{\mathrm{s}}$ (b), de la cambrure de la houle $\mathrm{H}_{\mathrm{s}} / \mathrm{L}$ (c) et du flux d'énergie $\mathrm{P}(\mathrm{d})$. Les barres verticales signalent les dates des levés topographiques.

Times series of $\mathrm{H}_{5}(\mathrm{a}), \mathrm{T}_{\mathrm{s}}(\mathrm{b})$, wave steepness $\mathrm{H}_{\mathrm{s}} / \mathrm{L}(\mathrm{c})$, and wave power $\mathrm{P}(\mathrm{d})$. Vertical lines indicate topographic survey dates.

pour permettre leur comparaison avec la topographie de la plage d'Omaha beach.

Le suivi morphologique de la plage concerne une zone d'environ $400 \mathrm{~m}$ de longueur (longshore) sur $400 \mathrm{~m}$ de largeur (cross-shore), couvrant l'estran du pied de dune jusqu'au niveau des basses mers de vives-eaux. Cette zone d'étude est située en dehors de tout aménagement en haute plage. Les levés topographiques sont réalisés à l'aide d'un DGPS centimétrique (Trimble 4400 rs) tracté sur la plage selon une maille régulière constituée de profils transversaux espacés de $10 \mathrm{~m}$. Une interpolation linéaire est effectuée pour réaliser un Modèle numérique de terrain (MNT) de maille rectangulaire de $5 \mathrm{~m}$ (longshore) $\times 2 \mathrm{~m}$ (cross-shore). D'avril 1999 à décembre 2000, 22 MNT comparables, dont deux exemples sont représentés dans la Fig. 2 ont été réalisés suivant un pas de temps d'environ un mois avec quelques levés intermédiaires. La précision altimétrique $( \pm 0,02 \mathrm{~m})$ est estimée à partir du levé régulier de points topographiques de référence situés sur des bornes fixes implantées sur la haute plage.

Les mesures des conditions d'agitation sont obtenues à l'aide d'un courantomètre houlographe (S4DW Interocean) situé aux niveaux des basses mers des grandes vives-eaux. Pour des raisons d'autonomie de la mémoire de l'appareil, les mesures sont réalisées selon des séquences de $9 \mathrm{~min}$ toutes les 4 heures. Les caractéristiques de la houle sont alors déduites d'une analyse spectrale du signal à haute fréquence des fluctuations de vitesses et de pressions. Elle est réalisée sur un échantillon de 1024 valeurs cadencées à $2 \mathrm{~Hz}$ sur une bande de fréquence allant de 0,05 à $0,33 \mathrm{~Hz}$. Ces coupures correspondent à la bande de fréquence des oscillations considérées comme relevant des vagues incidentes (3-20 s). La limite supérieure élimine les oscillations à haute fréquence dues au bruit électronique. La valeur inférieure fixée à $0,05 \mathrm{~Hz}$ employée communément dans les mers semifermées, à la limite basse du domaine des ondes infragravitaires (20-200 s) (Kroon, 1994). Les caractéristiques de l'agitation concernent la hauteur significative $\mathrm{H}_{\mathrm{s}}(\mathrm{m})$, la période significative $\mathrm{T}_{\mathrm{s}}(\mathrm{s})$, l'angle d'incidence de la houle au trait de côte $\theta\left({ }^{\circ}\right)$ et la hauteur d'eau h $(\mathrm{m})$. La longueur d'onde de la houle est déterminée à partir des paramètres $T_{\mathrm{s}}$ et h suivant la méthode de Hunt (1979). Les séries chronologiques des conditions d'agitation sur la basse plage pendant les deux années de mesures sont illustrées sur la Fig. 4. Le flux moyen d'énergie $\mathrm{P}$ (en Joules par seconde, $\mathrm{J} \mathrm{s}^{-1}$ ), transmis par crête de vagues et par unité de longueur dans le sens de propagation de la houle, est calculé pour chaque valeur discrète de la manière suivante (Hardisty, 1990) :

$$
P=\frac{1}{8} \rho_{w} \quad g H_{s}^{2}\left(\frac{L}{T}\right)\left[0,5\left(1+\frac{4 \frac{\pi}{L} h}{\sinh \left(4 \frac{\pi}{L} h\right)}\right)\right]
$$

avec $\rho_{\mathrm{w}}$, la masse volumique de l'eau de mer $\left(1027 \mathrm{~kg} \mathrm{~m}^{-3}\right)$, $\mathrm{g}$, l'accélération de la pesanteur $\left(9,81 \mathrm{~m} \mathrm{~s}^{-2}\right), \mathrm{H}_{\mathrm{s}}$, la hauteur 


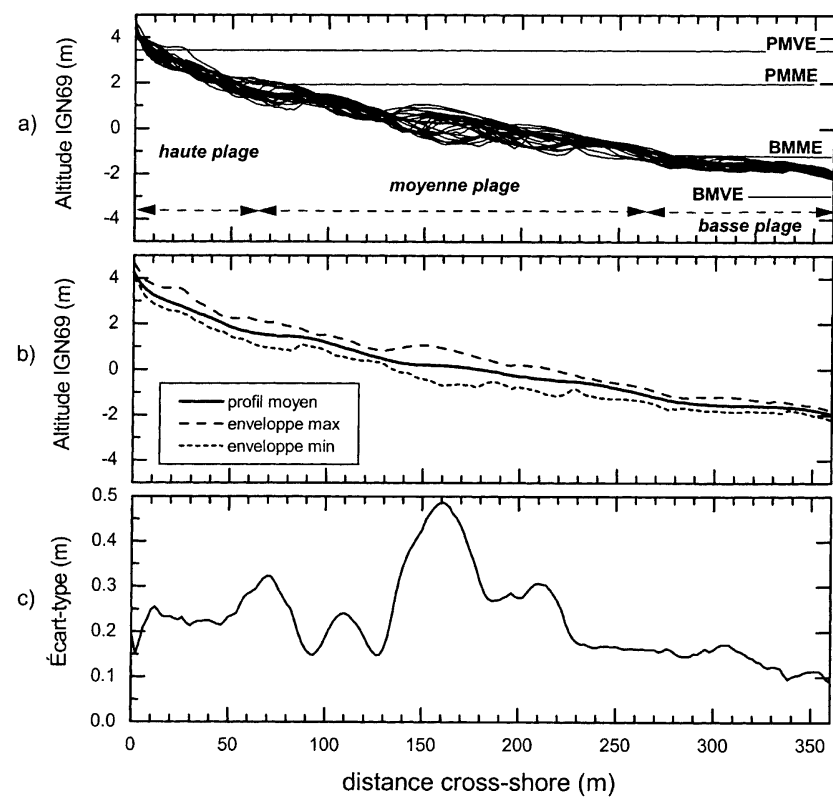

Fig. 5. Variabilité morphologique du profil central durant la période d'étude. a) superposition de tous les profils; b) profil moyen et enveloppe des profils ; c) écart-type des altitudes.

Morphological variability of the main profile throughout the study period. a) plot of all profiles over the study period ; b) mean profile and profile bundle ; c) standart deviation of elevation.

significative de la houle (m), L, la longueur d'onde de la houle (m), T (s), la période de la houle et $\mathrm{h}$, la hauteur d'eau (m).

\section{3. Évolution morphologique de la plage intertidale}

Elle est approchée en suivant l'évolution du profil de plage (2D) situé au centre de la zone d'étude. Après visualisation de l'ensemble des Modèles Numériques de Terrain tridimensionnels, ce profil central est considéré comme représentatif de l'évolution générale de la plage à l'échelle de temps mensuelle, bien que des changements morphologiques parallèlement au trait de côte (3D) peuvent en affecter localement la topographie.

\subsection{Variabilité morphologique du profil de plage}

Les principales données statistiques concernant l'évolution du profil central, sont représentées sur la Fig. 5 Elles sont déterminées à partir de l'ensemble des levés superposés Fig. 5a. L'altitude, en cote IGN69, est exprimée en fonction de la distance cross-shore. L'origine $\mathrm{x}=0$ situe la position du pied de dune. Vue la variabilité temporelle de la topographie intertidale, la largeur moyenne de chaque portion du profil (haute, moyenne et basse plage) est déterminée par commodité à partir de l'intersection du profil moyen calculé et des niveaux moyens de marées de vives-eaux et mortes-eaux. Ainsi, sur le profil d'Omaha, la haute plage est située entre 10 et $50 \mathrm{~m}$ du pied de dune, la moyenne plage entre 50 et $270 \mathrm{~m}$ et la basse plage entre 270 et $360 \mathrm{~m}$. La moyenne plage est divisée également en moyenne plage supérieure (50 à 135 m) et inférieure (135 à $270 \mathrm{~m}$ ) situées de part et d'autre du niveau moyen de la mer (0,35 m IGN69).

L'enveloppe des profils Fig. 5b met en évidence l'amplitude maximale des évolutions topographiques au cours de la période de suivi. Les plus importantes se situent sur la moyenne plage, où elles atteignent 1,60 m. Elles décroissent vers le large et deviennent minimales sur la basse plage. Sur la haute plage, l'amplitude d'évolution topographique est significative (environ $1 \mathrm{~m}$ ) mais reste inférieure à celle observée sur la moyenne plage. Le profil moyen qui intègre la grande variabilité temporelle des évolutions altimétriques, montre cependant des morphologies de barres atténuées sur la moyenne et basse plage Fig. 5b. La distribution de l'écart type des altitudes en fonction de la distance cross-shore Fig. 5c permet d'estimer le degré de mobilité des diverses zones de l'estran. La moyenne plage présente la plus grande variabilité, alors que celle-ci est inférieure sur la haute plage et décroît sur la basse plage, où l'écart type atteint $0,10 \mathrm{~m}$ à l'extrémité du profil.

Ces résultats montrent que la moyenne plage est la zone la plus réactive, en subissant les plus grandes évolutions topographiques par construction et destruction des systèmes de barres. Sur la basse plage, où les évolutions sont de beaucoup plus faible amplitude, une barre se maintient sur la totalité de la période de suivi.

\section{2. Évolution temporelle du profil central}

L'analyse de l'évolution topographique du profil central rend compte de la mobilité des barres ainsi que de leur mode de construction et de destruction. La Fig. 6 présente la totalité des profils centraux levés entre février 1999 et novembre 2000.

À cette échelle de temps, l'examen des différents profils met en évidence une hétérogénéité morphologique des différentes barres d'estran en fonction de leur position sur le profil. En effet, la basse plage est presque toujours marquée par une morphologie de barre, parfois très atténuée (juin 2000). La moyenne plage peut présenter des barres en nombre variable (1 à 3). Celles-ci peuvent être absentes sur sa partie supérieure (novembre 1999), alors qu'une barre est généralement présente sur sa partie inférieure. Sur la haute plage, une barre de swash, de plus faible amplitude et souvent très asymétrique, avec une pente orientée vers la dune plus forte que celle orientée vers la mer, peut parfois être observée (juin 1999, juin, juillet, septembre 2000). L'amplitude des barres est nettement supérieure sur la moyenne plage où elle peut atteindre $1 \mathrm{~m}$ (mars 2000, $\mathrm{x}=175 \mathrm{~m})$.

Trois séquences particulières, intégrant chacune une phase de construction (PC) et une phase de destruction (PD), peuvent être identifiées pendant la période de suivi.

La première séquence s'étend d'avril à novembre 1999. La phase de construction (PC1) d'avril à juillet 1999, se caractérise par le façonnement de quatre barres bien individualisées et leur migration vers le haut de plage. Entre juillet et octobre 1999, le profil conserve quatre barres mais leur 


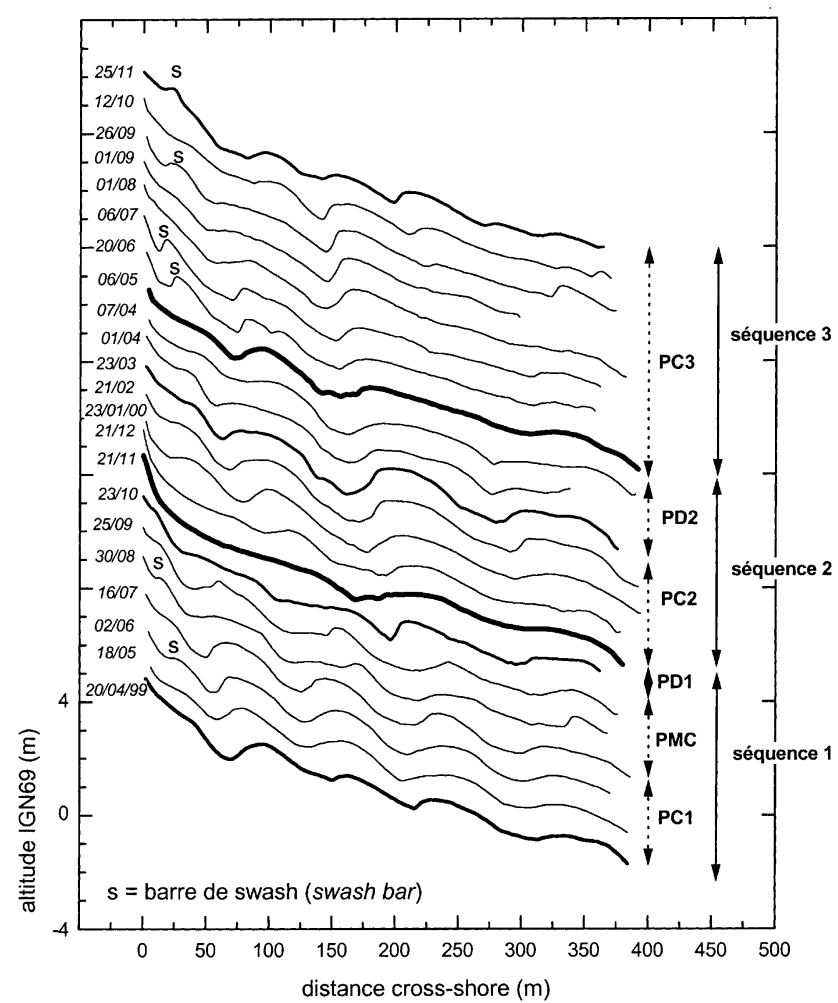

Fig. 6. Évolution temporelle du profil central. Les limites des séquences sont notées en gras et les phases intermédiaires en traits fins. $(\mathrm{PC}=$ Phase de construction, $\mathrm{PD}=$ Phase de Destruction, $\mathrm{PMC}=$ Phase de Migration des Chenaux).

Temporal evolution of the main profile. Sequence boundaries are in bold lines and intermediate states in thin lines. $(\mathrm{PC}=$ Stage of Construction, $\mathrm{PD}=$ Stage of Destruction, $\mathrm{PMC}=$ Stage of Channels Migration).

forme est fortement perturbée. Cette évolution traduit une déstabilisation morphologique locale due à la mise en place d'un système de chenaux de vidange au droit du profil central. Cette phase d'évolution 3D, observée à partir des MNT, correspond à une phase de migration des chenaux (notée PMC). L'évolution terminale de la séquence 1 est marquée, entre octobre et novembre 1999, par un lissage complet des barres sur la haute plage et la moyenne plage supérieure alors que l'on constate une stabilité des barres de la moyenne plage inférieure et de la basse plage (phase de destruction PD1).

La seconde séquence s'étend de novembre 1999 à mai 2000. Jusqu'en mars 2000, une barre se reforme sur la partie lissée du profil, alors que les barres déjà existantes s'exhaussent et migrent légèrement vers la haute plage (phase de construction PC2). Les levés entre le 23 mars et le 6 mai 2000 montrent une érosion de la haute plage et un aplanissement des barres, sur le moyen et bas estran (PD2).

La troisième séquence s'étend de mai 2000 à novembre 2000 et ne comprend qu'une phase de construction (PC3). Les levés s'arrêtant en novembre 2000, la séquence complète n'a sans doute pas été échantillonnée. Néanmoins, elle aboutit en début d'hiver au développement de cinq barres sur le profil de plage. Cette construction s'effectue par une succession d'états transitoires, fonction de réajustements morphologiques mineurs. Ainsi, de mai à juillet 2000, la basse plage

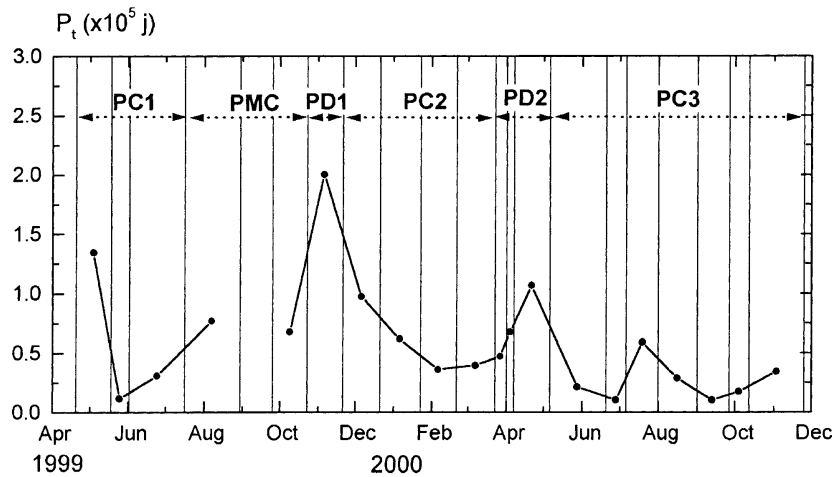

Fig. 7. Flux d'énergie total par période $\mathrm{P}_{\mathrm{t}}$ et comparaison avec les séquences morphodynamiques. Les barres verticales signalent les dates des levés topographiques.

Total wave power $\mathrm{P}_{\mathrm{t}}$ integrated over each period and comparison with morphodynamics sequences. Vertical lines indicate topographic survey dates.

reste stable, la barre de moyenne plage devient très asymétrique et une barre de swash se forme sur la haute plage. En août 2000 , les barres de haute plage et de moyenne plage supérieure sont lissées et le bas de plage ne présente pas d'évolution significative. De août 2000 à novembre 2000, les barres évoluent progressivement, une barre se forme sur la moyenne plage $(\mathrm{x}=225 \mathrm{~m})$ à partir du 26 septembre, ainsi que sur la haute plage, entre le $1^{\text {er }}$ et le 26 septembre.

D'un point de vue morphodynamique, les vitesses de migration des barres, calculées à partir du déplacement des crêtes, sont très différentes en fonction de leur position sur l'estran. Cela est remarquable lorsque les barres sont construites, individualisées et migrent vers le haut de la plage. Lors des phases PC1 et PC2, la vitesse de migration de la barre de basse plage est de l'ordre de $0,1 \mathrm{~m} \mathrm{j}^{-1}$. Sur la moyenne plage, elle atteint des vitesses de l'ordre de 0,1 à $0,4 \mathrm{~m} \mathrm{j}^{-1}$, alors que la barre de swash peut migrer vers la dune à une vitesse de $0,5 \mathrm{~m} \mathrm{j}^{-1}$ (juin-juillet 2000). De plus, le degré d'asymétrie des barres augmente du bas de plage vers le haut de plage, notamment lors des phases de construction.

\section{4. Évolution morphodynamique}

\subsection{Relations agitation - réponse morphologique}

Afin de quantifier précisément l'agitation au cours du temps, le flux d'énergie total par période $\mathrm{P}_{\mathrm{t}}$ (en Joules, J) est calculé par intégration sur le temps de $\mathrm{P}\left(\mathrm{J} \mathrm{s}^{-1}\right)$ entre deux levés topographiques consécutifs :

$$
P_{t}=\int_{1}^{2} P d t
$$

Ainsi, chaque période entre deux états morphologiques est caractérisée par un flux d'énergie prenant en compte l'ensemble des évènements hydrodynamiques qui se sont succédés entre les deux levés consécutifs (Fig. 7). Pour chaque phase de construction et de destruction précédemment dé- 
Tableau 1

Mise en relation du taux journalier d'énergie $P$ et du type de phase. Les points d'interrogations signalent un manque de données en raison d'un dysfonctionnement technique du houlographe

Relation between wave power daily rate $P$ and type of phase. The question marks indicate gap in the data due to technical dyfunction of the wave recorder

\begin{tabular}{|c|c|c|c|c|c|c|}
\hline Séquence & Phase & Début & Fin & $\mathrm{P}(\mathrm{J})$ & Nombre de jours & $\langle\mathrm{P}\rangle\left(\mathrm{J} \mathrm{j}^{-1}\right)$ \\
\hline \multirow[t]{4}{*}{1} & $\mathrm{PC} 1$ & $20 / 04 / 99$ & $16 / 07 / 99$ & 177224 & 87 & 2037 \\
\hline & PMC & $16 / 07 / 992$ & & $?$ & 99 & $?$ \\
\hline & PD1 & $23 / 10 / 992$ & & 200162 & 29 & 6902 \\
\hline & $\mathrm{PC} 2$ & $21 / 11 / 992$ & & 234751 & 123 & 1909 \\
\hline 2 & PD2 & $23 / 03 / 00$ & $06 / 05 / 00$ & 222086 & 44 & 5047 \\
\hline 3 & PC3 & $06 / 05 / 00$ & $25 / 11 / 00$ & 182186 & 203 & 897 \\
\hline
\end{tabular}

crite et identifiée selon des critères d'évolution morphologique, le flux total d'énergie incidente déterminé correspond à la somme du flux d'énergie des périodes de temps $\mathrm{P}_{\mathrm{t}}$ constituant la phase. Ce flux total est ensuite divisé par le nombre de jours de chaque phase, pour obtenir un taux journalier d'énergie $P$ (en Joules par jour, $\mathrm{J} \mathrm{j}^{-1}$ ) capable de la caractériser d'un point de vue dynamique :

$$
<P>=\frac{\sum P_{t}}{N}
$$

avec $\mathrm{P}_{\mathrm{t}}$, énergie totale par période (en Joules) et $\mathrm{N}$, nombre de jours constituant la phase. Les valeurs de $P$ pour chaque phase sont regroupées dans le Tableau 1. Les valeurs minimales sont associées aux phases de construction morphologique, elles sont égales ou inférieures à $2000 \mathrm{~J} \mathrm{j}^{-1}$. Lors des phases de destruction, $P$ est supérieur à $5000 \mathrm{~J} \mathrm{j}^{-1}$. Deux principaux modes d'évolution sont donc mis en évidence en fonction des caractéristiques morphologiques et dynamiques.

\subsection{1. Évolution lente et progressive en conditions de faible énergie}

Elle se traduit par une construction de 3 à 4 barres intertidales qui deviennent de plus en plus asymétriques. Elles se déplacent vers le haut de plage avec des vitesses de migration qui dépendent de leur position sur l'estran : $0,1 \mathrm{~m} \mathrm{j}^{-1}$ en bas de plage, jusqu'à $0,4 \mathrm{~m} \mathrm{j}^{-1}$ en haut de plage. De telles conditions sont également favorables au développement d'une barre de swash sur la haute plage souvent très asymétrique et qui migre vers la dune à des vitesses de l'ordre de $0,5 \mathrm{~m} \mathrm{j}^{-1}$. Ces conditions d'énergie résultent de périodes d'agitation faible avec des $\mathrm{H}_{\mathrm{s}}<1 \mathrm{~m}$ et des valeurs de cambrure des vagues $\mathrm{H}_{\mathrm{s}} / \mathrm{L}<0,04$ sur la basse plage Fig. 4 . La construction des barres est donc un processus progressif qui s'inscrit sur une période de temps relativement longue de plusieurs mois. Une morphologie convexe est initiée par une accumulation sableuse, puis la forme gagne en amplitude et migre simultanément vers le haut de plage.

\subsection{2. Évolution rapide en conditions de forte énergie}

Ces évolutions marquent la réponse du profil de plage à une augmentation globale du niveau d'énergie des houles $\left(\mathrm{H}_{\mathrm{s}}>1 \mathrm{~m}\right.$ et $\left.\mathrm{H}_{\mathrm{s}} / \mathrm{L}>0,04\right)$. L'impact des tempêtes sur la morphologie est très variable. Une tempête isolée comme celle de début mai 1999 n'affecte que très peu les barres intertidales. En revanche, le passage d'une succession de tempêtes peut détruire complètement les barres de moyenne et haute plage (PD1). Les barres situées plus bas peuvent être lissées mais restent identifiables, elles peuvent également subir des érosions partielles sous l'effet de tempêtes de fortes magnitudes (PD2). Une telle diversité des réponses morphologiques du profil de plage met en évidence la complexité des processus morphodynamiques qui interagissent lors des tempêtes.

\subsection{Relations évolution morphodynamique - évolution $d u$ stock sédimentaire intertidal}

Les barres sableuses sont généralement reconnues comme des zones de stockage dans l'analyse du budget sédimentaire des côtes sableuses. L'étude de l'évolution du stock sableux d'Omaha beach a pour but d'évaluer les relations entre les changements morphologiques (formation/destruction de barres) et l'évolution du volume sédimentaire de la plage intertidale. Pour chaque levé, les volumes sont calculés par cubature des Modèles Numériques de Terrain en trois dimensions. L'incertitude des calculs est évaluée à $\pm 2640 \mathrm{~m}^{3}$ à partir de la précision de la mesure topographique $( \pm 0,02 \mathrm{~m})$ et de l'aire de la zone de comparaison de l'ensemble des levés (400 $\times 330=132000 \mathrm{~m}^{2}$ ).

L'évolution du volume total de la plage par rapport au volume initial (17/02/1999) est représentée sur la Fig. 8 D'avril à décembre 1999, le volume reste relativement stable, au regard de la précision des mesures. Une brutale augmentation $\left(+8000 \mathrm{~m}^{3}\right)$ se produit entre décembre 1999 et février 2000. Par la suite, le budget décroît entre février et mai 2000, puis reste stable jusqu'au mois de décembre 2000. À l'échelle des séquences morphodynamiques, cette évolution est modulée par des fluctuations, positives pendant les phases de construction et négatives, lors des séquences de destruction. Toutefois, les fluctuations sont de l'ordre de grandeur de l'incertitude de mesure, la séquence PC2 (novembre 1999-mars 2000) étant une exception.

Pour chaque phase, l'amplitude des évolutions du volume sédimentaire est mise en relation avec le taux journalier d'énergie $P$ Fig. 9]. Les accrétions significatives de la plage sont reliées à des conditions d'énergie modérée $\left(P \approx 2000 \mathrm{~J} \mathrm{j}^{-1}\right)$. En deçà de ce taux d'énergie, l'accrétion est faible à nulle. Au-delà, les réponses volumétriques sont variables. Des tempêtes de forte intensité (PD2 : mars- 


\section{différence de volume $\left(\mathrm{m}^{3}\right)$}

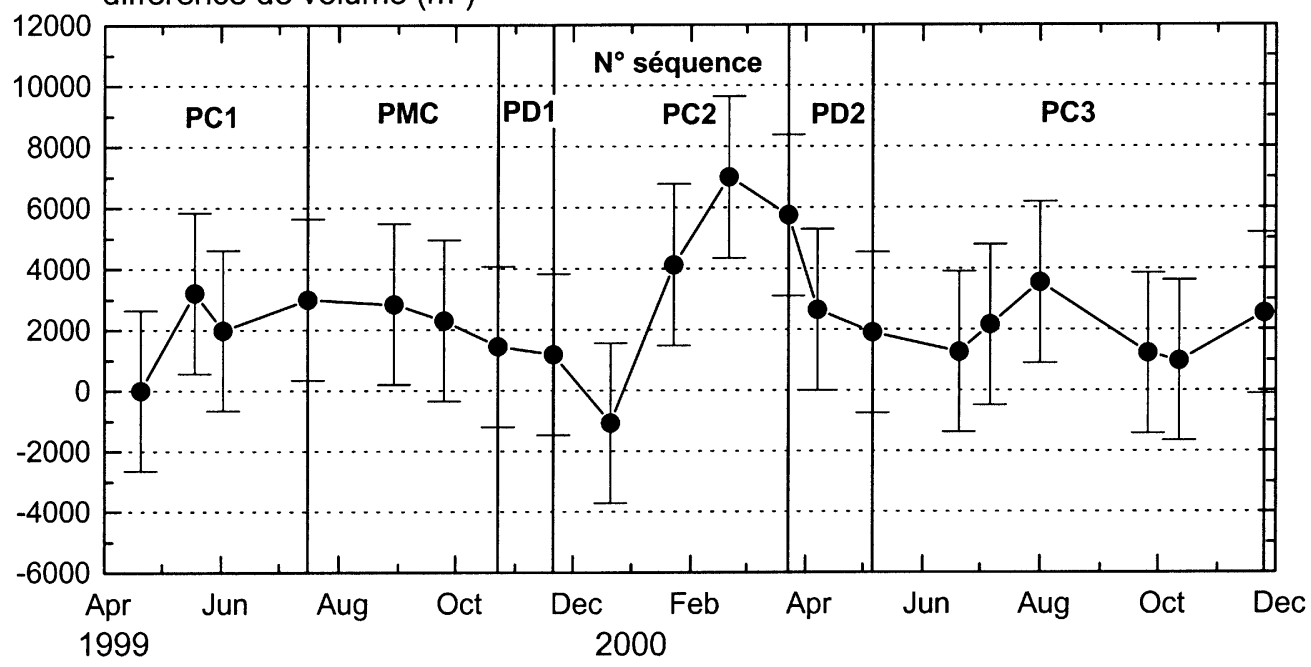

Fig. 8. Évolution du volume sédimentaire total de la plage d'avril 1999 à novembre 2000.

Evolution of beach sand volume from April 1999 to November 2000.

mai 2000) entraînent une érosion significative de l'estran $\left(-4000 \mathrm{~m}^{3}\right)$ alors que le volume est plutôt stable lors des périodes énergétiques maximales (PD1). Compte tenu de la précision des mesures $\left( \pm 2700 \mathrm{~m}^{3}\right)$, ces observations montrent que l'impact de l'agitation du plan d'eau reste relativement limité sur le volume sableux stocké dans la zone intertidale. Ceci tend à montrer que les barres intertidales de la plage d'Omaha beach sont la traduction topographique de redistributions locales d'un stock sédimentaire intertidal stable sur la période de suivi. D'après nos mesures, les conditions optimales d'accrétion de barres sableuses sont celles correspondant à un taux d'énergie $P$ d'environ $2000 \mathrm{~J} \mathrm{j}^{-1}$.

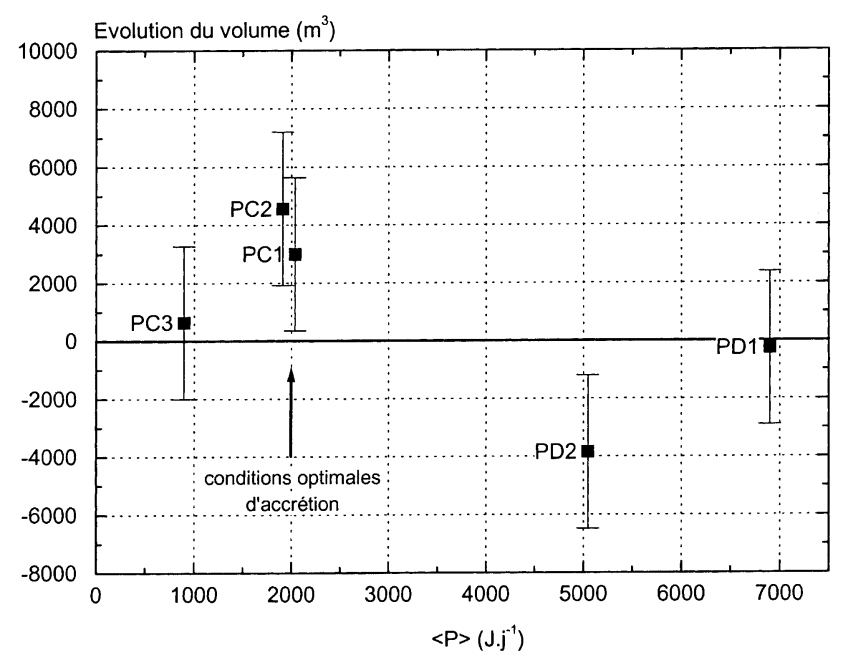

Fig. 9. Relation entre le taux journalier d'énergie $P$ et l'évolution du volume pour chaque phase.

Relation between wave power daily rate $P$ and volumetric evolution for each stage.

\section{Discussion}

Le suivi morphologique et hydrodynamique d'Omaha beach pendant deux années, apporte des indications sur la morphodynamique d'une plage macrotidale à barres à moyen terme.

1. L'examen de critères morphodynamiques permet d'identifier deux types de barres intertidales :

o Les barres de haute plage sont des formes éphémères, asymétriques, très mobiles et contemporaines de longues périodes d'agitation faible. Des barres similaires sont communément observées sur les plages microtidales (Davis et Fox, 1972 ; Dabrio et Polo, 1981 ; Dabrio, 1982 ; Sunamura et Takeda, 1984), ainsi que sur les hauts estrans des plages méso- et macrotidales (Wright et al., 1982 ; Michel et Howa, 1999). Elles correspondent à la définition de swash bar (barre de swash) de Greenwood et Davidson-Arnott (1978) et Orford et Wright (1978). Leur mode de formation est principalement dû à l'action du swash en conditions de faible énergie de la houle. Les transports sédimentaires sont très dissymétriques entre les phases d'uprush (jet de rive) et de backwash (nappe de retrait), en raison de l'infiltration de l'eau dans le sable sous-saturé (Duncan, 1964). Degryse et al (2002) discutent l'importance de la pente, de la localisation de la nappe phréatique et des oscillations infragravitaires de la surface libre, sur la formation de ces barres de swash. En contexte tidal, la migration vers le haut de plage est due au dépassement chronique de la crête de barre par les niveaux de pleines mers en revif (Kroon, 1994 ; Degryse et al., 2002). Les événements de haute énergie en vives-eaux entraînent généralement leur destruction complète. 
O Les barres présentes sur les moyenne et basse plages sont des morphologies plus stables que les précédentes. À l'échelle de temps du suivi, leur formation, synchrone de périodes de faible énergie de la houle, s'accompagne d'une migration vers le haut de plage de l'ordre de 0,1 à $0,4 \mathrm{~m} \mathrm{j}^{-1}$. Ces barres sont conformes à la définition de ridges and runnels stricto sensu (King et Williams, 1949 ; King, 1972 ; Orford et Wright, 1978). Ce sont des formes dont la résistance au passage des tempêtes est fonction de leur position sur l'estran, les plus basses étant les plus stables, ce qui confirme les observations de Mulrennan (1992) sur les côtes irlandaises et leur qualification de « semi-permanentes ».

2. L'évolution morphologique de la plage d'Omaha est complexe et rythmée par une alternance de conditions calmes, sans vent ou vents de terre, qui favorisent l'accrétion des barres et de périodes de forte énergie, lors des tempêtes de NW à NE, qui déstabilisent la morphologie antécédente.

- Les ordres de grandeurs des temps de construction et des vitesses de migration des barres vers le haut de plage sont en accord avec les études portant sur des plages similaires soumises à des conditions comparables (King et Williams, 1949 ; Levoy et al., 1998, Voulgaris et al., 1998). Sur la base des expérimentations réalisées et des conditions météorologiques présentées, ces auteurs attribuent la mobilité des barres au transport sédimentaire dans la zone de swash au cours de son balayage de l'estran par la marée. Stépanian et al. (2001) montrent que la migration est le résultat d'une redistribution locale des sédiments à l'échelle de la barre, du revers marin vers le talus orienté vers la dune, ce qui participe à l'augmentation de l'asymétrie des barres. La différence spatiale des vitesses de migration traduit l'amplification des effets du swash du bas vers le haut de plage, due à un renforcement de l'énergie des houles sur la partie haute et moyenne du profil au cours du cycle tidal (Wright et al., 1982 ; Masselink, 1993 ; Masselink et Hegge, 1995 ; Levoy et al., 2001).

- Les modalités de la construction des barres sont également mises en évidence. Elles comportent une phase d'initiation de la morphologie de barre, suivie d'une augmentation de l'asymétrie accompagnée d'une migration de la crête vers le haut de plage. La localisation de l'initiation des barres reste imprécise. Le rôle de la morphologie antécédente semble déterminant sur ce point, à la manière des rétroactions et interactions morphodynamiques complexes entres les différentes barres subtidales, décrites par Lee et al. (1998), Ruessink et Terwindt (2000), sur les plages micro- et mésotidales américaines (Duck, Caroline du Nord) et hollandaises (Terschelling).

- Les réponses morphologiques du profil de plage à l'intensification de l'énergie de la houle sont variables.
Les évolutions vont de la stabilité du bas estran à la destruction complète des barres sur la moyenne plage supérieure et la haute plage. Les suivis réalisés sur Omaha beach durant deux années permettent d'isoler clairement ces phases de destruction. Chacune de ces périodes $\left(\langle P\rangle>5000 \mathrm{~J} \mathrm{j}^{-1}\right)$ est caractérisée sur la basse plage par des épisodes aux $\mathrm{H}_{\mathrm{s}}$ dépassant $1 \mathrm{~m}$ et une cambrure $\left(\mathrm{H}_{\mathrm{s}} / \mathrm{L}\right)$ supérieure à 0,04 . Le taux journalier d'énergie $\langle P\rangle$ est un paramètre discriminant les phases constructives et destructives à l'échelle de temps du suivi. La valeur seuil, supérieure à $2000 \mathrm{~J}$, entre ces phases reste imprécise en raison de la complexité naturelle des processus qui interagissent. Ceux-ci concernent à la fois les conditions de forçages hydrodynamiques et les caractéristiques morphologiques de la plage précédant la tempête. Il est admis que l'intensification du courant de retour, en relation avec une surcote lors des tempêtes, est le principal facteur d'érosion des barres tant intertidales (Kroon, 1994) que subtidales (Ruessink et Terwindt, 2000). Les caractéristiques propres des tempêtes (intensité, durée, obliquité de la houle) sont donc à prendre en compte, ainsi que la succession des tempêtes au cours du temps. En effet, la forme du profil peut être mise à l'équilibre par une première tempête et rester stable lors du passage des suivantes (Lee et al., 1998). De plus, le niveau de la marée et la position de l'évènement par rapport au cycle tidal lunaire sont primordiaux sur le niveau d'attaque de l'estran par les vagues (Levoy et al., 2001). Ainsi, un épisode de tempête en mortes-eaux va induire des changements morphologiques plus importants sur la moyenne et basse plage (mars-avril 2000) qu'une tempête en vives-eaux dont l'impact sur la haute plage est facilement identifiable (novembre 1999). De son côté, la morphologie initiale exerce un contrôle sur la dissipation de l'énergie des vagues sur le profil : les barres constituent des filtres énergétiques qui induisent prématurément le déferlement des plus grosses vagues et laissent se propager les vagues de hauteur inférieure (Carter et Balsillie, 1983). Ce caractère dissipatif est d'autant plus important que les barres sont nombreuses et de forte amplitude, ce qui a pour effet d'entretenir leur inertie morphodynamique et leur résistance aux tempêtes. Sur Omaha beach, celles-ci sont fragilisées par les hétérogénéités longshore de la morphologie, perturbant la continuité latérale des barres. En effet, la phase de destruction PC2 (novembre 1999) montre que l'érosion complète des barres de la partie supérieure de la plage est précédée par une phase de déstabilisation (PMC) due à la mise en place de chenaux de vidange au niveau du profil suivi. Cette phase montre que la dynamique des chenaux est un facteur supplémentaire à intégrer aux processus de destruction des barres intertidales.

3. Sur Omaha beach, la construction des barres correspond à une réorganisation morphologique d'un stock 
sédimentaire intertidal stable à l'échelle du suivi, mais dont les relations avec le domaine subtidal restent à préciser. Celles-ci sont probablement très limitées compte tenu des changements altimétiques réduits observés à l'extrémité du profil de plage étudié (Fig. 5). La dynamique des formes intertidales est la conséquence d'une redistribution locale des sédiments, tant en conditions d'accrétion, du revers marin vers le talus, qu'en conditions de destruction, les sédiments érodés des barres, notamment de la haute et moyenne plage, comblant les bâches les plus proches.

\section{Conclusions}

Cette étude montre que le comportement morphodynamique de la plage macrotidale d'Omaha beach est séquentiel. En effet, l'évolution de la topographie intertidale est rythmée par des phases de construction et de destruction des barres sableuses. Ces phases traduisent des réorganisations du stock sédimentaire intertidal, dont le volume est stable à moyen terme, en réponse aux variations du forçage hydrodynamique observées à court-terme. Ainsi, les phases de construction des barres résultent de longues périodes d'agitation faible $\left(\mathrm{H}_{\mathrm{s}}<1 \mathrm{~m}\right)$, alors que leur destruction découle d'une augmentation rapide de la hauteur des houles $\left(\mathrm{H}_{\mathrm{s}}>1 \mathrm{~m}\right)$ lors des tempêtes. Ces épisodes de courte durée sont, bien entendu, les facteurs principaux responsables de l'érosion des barres. Ils correspondent à une intensification des processus hydrodynamiques (courants orbitaux, courant de retour, courants de compensation). L'ensemble des interactions entre ces processus morphodynamiques aboutit à la création d'un nouvel état morphologique à partir duquel les barres se développent durant les périodes de faible énergie. La dynamique des chenaux de drainage de l'estran est également mise en évidence. Elle est à l'origine d'une déstabilisation morphologique locale des barres. En effet, l'apparition de ces chenaux et leur migration longshore dans un contexte d'agitation modérée, incisent les reliefs intertidaux, amorçant leur destruction, souvent complètement effective lors des tempêtes suivantes. Ces zones topographiques basses canalisent alors les écoulements vers le large amplifiant leur rôle destructeur en période de forte agitation. Le réseau des chenaux de drainage est ainsi une composante morphologique tridimensionnelle générant une variabilité longitudinale du comportement morphodynamique des plages à barres et à bâches.

\section{Remerciements}

Ces travaux ont été financés dans le cadre du Programme National d'Environnement Côtier (PNEC) au sein de l'Action de Recherche Thématique ART7 « hydrodynamique sédimentaire des côtes sableuses ». Les auteurs remercient l'ensemble des personnes qui ont participé aux interventions sur le terrain et Méteofrance pour la fourniture des données météorologiques. Ils remercient également Mr le Professeur
Claude Larsonneur pour sa lecture critique lors de la préparation de cette note, ainsi que les rapporteurs dont les commentaires améliorent la lisibilité du manuscrit.

\section{Références}

Carter, R.W., Balsillie, J.H., 1983. A note on amount of wave energy transmitted over nearshore sand bars. Eart. Surf. Proc. Land. 8, 213-222.

Corbau, C., Howa, H., Tessier, B., de Resseguier, A., Chamley, H., 1994. Evaluation du transport sédimentaire sur une plage macrotidale par traçage fluorescent, Dunkerque Est, France. C.R. Acad. Sci. Paris 319 (II), 1503-1509.

Dabrio, C.J., Polo, M.D., 1981. Flow regime and bedforms in a ridge and runnel system. S.E. Spain. Sedim. Geol. 28, 97-110.

Dabrio, C.J., 1982. Sedimentary structures generated on the foreshore by migrating ridge and runnel systems on microtidal and mesotidal coasts of South Spain. Sedim. Geol. 32, 141-151.

Davis, R.A., Fox, W.T., 1972. Coastal processes and nearshore sand bars. J. Sed. Petrol. 42 (2), 401-412.

Degryse, C., Stepanian, A., Levoy, F., Monfort, O., 2002. Swash bar behavior on a macrotidal barred beach (en préparation). .

Duncan, J.R., 1964. The effects of watertable and tide cycle on swashbackwash sediment distribution and beach profile developpment. Mar. Geol. 2, 186-197.

Greenwood, B., Davidson-Arnott, R.G.D., 1978. Sedimentation and equilibrium in wave formed bars : a review and case study. Can. J. Earth Sci. $16,323-332$.

Hardisty, J., 1990. Beaches, Form and Process. Unwyn Hyman, London p. 324.

Hunt, J.N., 1979. Direct solution of wave dispersion equation. J. Wat. Por. Coast. Oce. Divi 4, ASCE, 457-459.

King, C.A.M., 1972. Beaches and Coasts. 2nd Ed. Edward Arnold, London p. 570 .

King, C.A.M., Williams, W.W., 1949. The formation and mouvement of sand bars by wave action. Geogr. J. 113, 70-85.

Kroon, A., 1994. Sediment transport and morphodynamics of the beach and nearshore zone, near Egmond, The Netherlands. Thèse de Doctorat, Univ. Utrecht, Hollande p. 275.

Lee, G., Nicholls, R.J., Birkemeier, W.A., 1998. Storm-driven variability of the beach-nearshore profile at Duck, North Carolina, USA, 1981-1991. Mar. Geol. 148, 163-177.

Levoy, F., Anthony, E., Barusseau, J.P., Howa, H., Tessier, B., 1998. Morphodynamique d'une plage macrotidale à barres. C.R. Acad. Sci. Paris 327, 811-818.

Levoy, F., Monfort, O., Larsonneur, C., 2001. Hydrodynamic variability on megatidal beaches, Normandy, France. Cont. Shelf. Res. 21, 563-586.

Masselink, G., 1993. Simulating the effects of tides on beach morphodynamics. J. Coast. Res. 15 (SI), 180-197.

Masselink, G., Anthony, E.J., 2001. Location and height of intertidal bars on macrotidal ridge and runnel beaches. Eart. Surf. Proc. Land. 26, 759-774.

Michel, D., Howa, H., 1999. Short-term morphodynamic response of a ridge and runnel system on a mesotidal beach. J. Coast. Res. 15 (2), 428-437.

Mulrennan, M.E., 1992. Ridge and runnel beach morphodynamics : an example from the central east coast of Ireland. J. Coast. Res. 8 (4), 906-918.

Orford, J.D., Wright, P., 1978. What's in a name? - Descriptive or genetic implications of «ridge and runnel» topography. Mar. Geol. 28, M1-M8.

Ruessink, B.G., Terwindt, J.H.J., 2000. The behaviour of nearshore bars on the time scale of years : a conceptual model. Mar. Geol. 163, 289-302.

Ruessink, B.G., Van Enckevort, I.M.G., Kingston, K.S., Davidson, M.A., 2000. Analysis of observed two- and three-dimensional nearshore bar behaviour. Mar. Geol. 169, 161-183.

Shand, R.D., Bailey, D.G., 1999. A review of net offshore bar migration with photographic illustrations from Wanganui, New Zealand. J. Coast. Res. 15 (2), 365-378. 
Shand, R.D., Bailey, D.G., Sherpherd, M.J., 1999. An inter-site comparison of net offshore bar migration characteristics and environmental conditions. J. Coast. Res. 15 (3), 750-765.

Short, A.D., 1999. Handbook of Beach and Shoreface Morphodynamics. Wiley, Chichester p. 379.

Simmonds, Voulgaris, G., Huntley, D.A., 1995. Dynamic processes on a ridge and runnel beach. Proceedings of the 2 th International Conference of Coastal Dynamics. ASCE, Gdansk, Poland, pp. 868-878.

Simmonds, D.J., O'Hare, T.J., Huntley, D.A., 1996. The influence of long waves on macrotidal beach morphology. Proceedings of the 25th International Conference of Coastal Engineering. ASCE, New York, pp. 3090-3103.
Stépanian, A., Vlaswinkel, B., Levoy, F., Larsonneur, C., 2001. Sediment transport on a macrotidal ridge and runnel beach during accretion conditions. Proceedings of the 4th International Conference of Coastal Dynamics. ASCE, Lund, Sweden, pp. 1017-1027.

Sunamura, T., Takeda, I., 1984. Landward migration of inner bars. Mar. Geol. 60, 63-78.

Voulgaris, G., Simmonds, D., Michel, D., Howa, H., Collins, M.B., Huntley, D.A., 1998. Measuring an modelling sediment transport on a macrotidal ridge and runnel beach: an intercomparaison. J. Coast. Res. 14 (1), 300-315.

Wright, L.D., Nielsen, P., Short, A.D., Green, M.O., 1982. Morphodynamics of a macrotidal beach. Mar. Geol. 50, 97-128. 